\title{
High Frequency Longitudinal Damped Vibrations of a Cylindrical Ultrasonic Transducer
}

\author{
Mihai Valentin Predoi, ${ }^{1}$ Cristian Cătălin Petre, ${ }^{2}$ Ovidiu Vasile, ${ }^{1}$ and Mihail Boiangiu1 \\ ${ }^{1}$ Department of Mechanics, Polytechnic University of Bucharest, Splaiul Independenţei 313, 60048 Bucharest, Romania \\ ${ }^{2}$ Department of Strength of Materials, University Politehnica of Bucharest, Splaiul Independenţei 313, 60048 Bucharest, Romania
}

Correspondence should be addressed to Mihai Valentin Predoi; predoi@gmail.com

Received 27 February 2014; Accepted 16 June 2014; Published 24 June 2014

Academic Editor: Xinjie Zhang

Copyright (c) 2014 Mihai Valentin Predoi et al. This is an open access article distributed under the Creative Commons Attribution License, which permits unrestricted use, distribution, and reproduction in any medium, provided the original work is properly cited.

Ultrasonic piezoelectric transducers used in classical nondestructive testing are producing in general longitudinal vibrations in the $\mathrm{MHz}$ range. A simple mechanical model of these transducers would be very useful for wave propagation numerical simulations, avoiding the existing complicated models in which the real components of the transducer are modeled by finite elements. The classical model for longitudinal vibrations is not adequate because the generated longitudinal wave is not dispersive, the velocity being the same at any frequency. We have adopted the Rayleigh-Bishop model, which avoids these limitations, even if it is not converging to the first but to the second exact longitudinal mode in an elastic rod, as obtained from the complicated PochhammerChree equations. Since real transducers have significant vibrations damping, we have introduced a damping term in the RayleighBishop model, increasing the imaginary part and keeping almost identical real part of the wavenumber. Common transducers produce amplitude modulated signals, completely attenuated after several periods. This can be modeled by two close frequencies, producing a "beat" phenomenon, superposed on the high damping. For this reason, we introduce a two-rod Rayleigh-Bishop model with damping. Agreement with measured normal velocity on the transducer free surface is encouraging for continuation of the research.

\section{Introduction}

Longitudinal vibrations of short rods are the basic phenomenon involved in most ultrasonic transducers, since shear wave transducers are seldom used. From the classical theory of longitudinal vibrations of rods to the exact dynamic elasticity equation of motion, all deal with undamped motions. However, the conflicting requirements of high amplitude resonance displacements and short signal duration used for feature detection are implying more elaborate mechanical models which include damping of the elastic motion.

A review of fundamental models for longitudinal vibrations of rods is given by Shatalov et al. [1] and Fedotov et al. [2]. The classical approximate theory of longitudinal vibration of rods was developed during the 18th century by J. D’Alembert, D. Bernoulli, L. Euler, and J. Lagrange. The classical theory is generally accepted due to its simplicity and is called a unimodal theory since only the fundamental symmetric guided mode is approximated. It is broadly used for the design of low frequency waveguides such as ultrasonic transducers and mechanical filters. Other unimodal theories are presented, such as the Rayleigh-Bishop theories, with two, three, or four terms in radial and longitudinal displacements fields or the Rayleigh-Love theory neglecting the radial stress. All these unimodal theories are compared against the exact Pochhammer-Chree frequency equation in the mentioned reference.

Many researchers have elaborated transducers models including the piezoelectric component, the backing layer, and casing [3]. The transient response of $2 \mathrm{MHz}, 12.7 \mathrm{~mm}$ diameter transducer is analyzed using an excitation signal of $2 \mathrm{MHz}$ sine-wave half cycle. The model was implemented using commercial software using the finite element method (FEM) by Medina et al. 
Coupled oscillator models of resonant transducers, finite element modeling issues in transducers and arrays were developed by Abboud et al. [4]. Accuracy of FEM analysis is dependent upon the accuracy of the dielectric, piezoelectric, and elastic properties used to represent the constituent materials of the model. The work of Ali [5] on a discrete time model of the impulse response of matched and backed piezoelectric transducers has led to the design of sensitive, short-response transducers. The model is obtained from the analysis of the electrical equivalent circuit of a piezoelectric device.

Guelaz et al. [6] present an ultrasonic piezoceramic transducer model integrated in a global measurement cell, a model dedicated to biological ultrasound characterization. Characterization of different mediums, like water and ethanol, by a comparison method is based on the ultrasonic parameter estimation. The good results obtained for temporal simulation and nonlinear parameter estimation compared to measurements and can be extended to multilayer characterization with an ultrasonic nonlinear imaging probe model.

The model used in [7] by Dragan et al. for the ultrasonic sandwich transducer is obtained by appropriate connecting of corresponding mechanical input ports of the transducer component parts, taking into account the actual existing contacts of the surfaces represented by these inputs. Under the condition that the outer contour surfaces of transducer component parts are loaded by surrounding environment acoustic impedances, which are small for the unloaded surface, and with alternating voltage applied to the electric inputs, it is possible to determine any transfer function of interest for the given system.

The FEM was proven by Nygren [8] to be a useful way of simulating piezoelectric ultrasonic transducers. The method is versatile as it can be used to emulate both one-dimensional models and more realistic three-dimensional transducer models. However, the suggested minimal requirement of 6 elements per wavelength would cause the solution of a complete three-dimensional model to become practically unattainable. Thankfully, many models can be significantly reduced in size, when mirror and axial symmetry is taken into consideration.

The work of Kocbach [9] presents an important basis for the first step of the design process of piezoelectric transducers. This has been done through an analysis of the radiated sound field, response functions, and vibration of the basic parts of piezoelectric disk transducers (the piezoelectric disk and the front layer) and an analysis of the influence of varying geometry and material parameters of the transducer on these quantities. The analysis has also been targeted at understanding the influence of the different design parameters of the simple piezoelectric transducer on the response functions of the transducer, in order to provide guidelines on how to vary the design parameters in the second step of the design process. The FEM has been used to simulate the $3 \mathrm{D}$ numerical models developed by Porto [10]. With the numerical optimization of new ultrasonic transducer designs in prospect, the computation time is a key problem. The different existing or developed optimization methods, absolutely valid with the correspondent analytical models, have shown their limitations. An optimization method based on genetic algorithms (GA) has been envisioned, thanks to its ease of programming and its intrinsic modularity. The developed and implemented new algorithms have been tested using many test functions and have shown satisfactory results. Consequently, new $2 \mathrm{D}$ models of ultrasonic transducers have been imagined and optimized, with accurate results and fast enough computations.

Schubert and Lamek [11] present the elastodynamic finite integration technique (EFIT) combined with point-sourcesynthesis (PSS) in order to efficiently calculate 3D ultrasonic wave fields in the time domain. The technique can be applied to arbitrarily layer locally isotropic media and includes the complete spectrum of linear elastic wave phenomena like surface and interface waves, head waves, mode conversion, and multiple scattering. The proposed technique is not restricted to axisymmetric isotropic problems since the basic point source data can also be calculated by using a single $3 \mathrm{D}$ calculation of an arbitrarily layered anisotropic medium.

An accurate simulation of the dynamic response of a longitudinal wave transducer requires parametric sweep of the damping characteristics. Damped longitudinal vibrations can be modeled in several ways. Pintelon et al. [12] used frequency dependent complex Young modulus, which can be can be experimentally determined and is capable of measuring low level of damping in the frequency domain. Wave propagation in viscoelastic structures, including rods, has also been investigated by Castaings et al. [13], using finite elements and complex moduli for the materials.

In the present work, a unimodal model for the longitudinal vibrations of a rod is investigated in the second section. The classical model for longitudinal vibrations is proved to be inadequate because the generated longitudinal wave is not dispersive, the velocity being the same at any frequency. The slightly more complicated Rayleigh-Love model is dispersive, but, beyond a critical frequency, the wave will not propagate. We have adopted the Rayleigh-Bishop model, which avoids these limitations, even if it is not converging to the first but to the second exact longitudinal mode in an elastic rod, which can be obtained from the complicated Pochhammer-Chree equations. Since real transducer has significant vibrations damping, we have introduced, in the third section, a damping term in the Rayleigh-Bishop model, proving negligible changes in the real part of the wavenumbers but significant frequency increasing imaginary parts absolute values. The real transducers produce an amplitude modulated signal, completely attenuated after several periods. Such behavior can be modeled by two close valued frequencies, producing a "beat" phenomenon, superposed on high damping. For this reason, we introduce a two-rod Rayleigh-Bishop model with damping in the fourth section. Agreement with measured normal velocity, shown in the fifth section, is encouraging.

\section{Comparison of Theoretical Models in the High Frequency Range}

The mechanical model considers a circular rod of length $L$, cross-section area $S$, mass density $\rho$, Young modulus $E$, and 


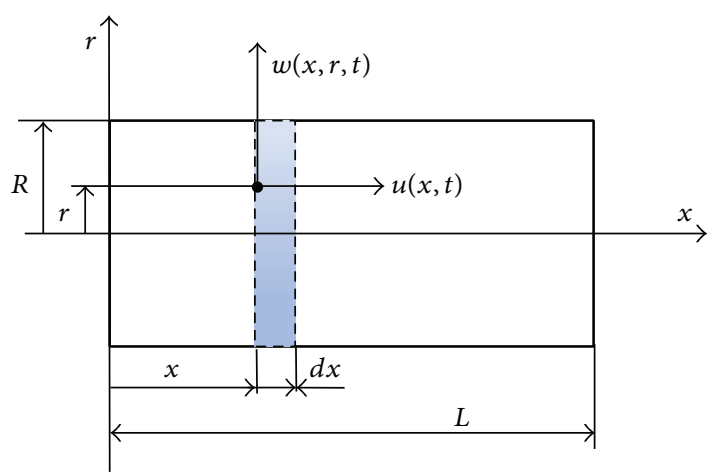

(a)

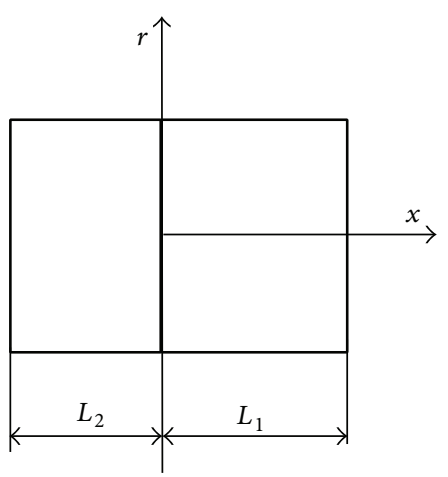

(b)

Figure 1: Geometry of the rod with local displacements in cylindrical frame (a). Two rods (b).

Poisson coefficient $v$. According to the Rayleigh-Love theory, the longitudinal vibrations of the rod defined in Figure 1 are described by the following displacements field:

$$
u(x, r, t)=u(x, t), \quad w(x, r, t)=-v r u_{x} .
$$

The radial displacement $w$ is proportional to the local radius $r(0 \leq r \leq R)$ in which $R$ is the outer radius of the rod and $u_{x}=\partial u(x, t) / \partial x$. The strains in cylindrical frame, using (1), are as follows:

$$
\begin{gathered}
\varepsilon_{x x}=u_{x_{x}} ; \quad \varepsilon_{r r}=w,_{r}=-v \mathcal{v},_{x} ; \\
\varepsilon_{x r}=u_{r_{r}}+w_{x_{x}}=-\nu \mathcal{v},_{x x} ; \quad \varepsilon_{\theta \theta}=\frac{w}{r}=-v u_{x_{x} .}
\end{gathered}
$$

The relevant stress components, using the Lamé constants $\lambda=$ $E v /(1-2 v)(1+\nu), \quad \mu=E / 2(1+\nu)$, are as follows:

$$
\begin{gathered}
\sigma_{x x}=(\lambda+2 \mu) \varepsilon_{x x}+\lambda\left(\varepsilon_{r r}+\varepsilon_{\theta \theta}\right)=E u_{x_{x}}, \\
\sigma_{x r}=\mu \varepsilon_{x r}=-\nu \mu r u_{x x} .
\end{gathered}
$$

It can be proven that the radial displacements defined cancel the radial stress:

$$
\begin{aligned}
\sigma_{r r} & =(\lambda+2 \mu) \varepsilon_{r r}+\lambda\left(\varepsilon_{x x}+e_{\theta \theta}\right) \\
& =-\nu(\lambda+2 \mu) u,_{x}+\lambda\left(u,_{x}+\frac{w}{r}\right) \\
& =-\nu(\lambda+2 \mu) u,_{x}+\lambda(1-\nu) u_{x_{x}}=0 .
\end{aligned}
$$

2.1. The Rayleigh-Love Model. The kinetic energy $(T)$ and the strain potential energy $(V)$ for this model are as follows:

$$
\begin{aligned}
T & =\int_{0}^{L} \frac{\rho}{2} \int_{S}\left(\dot{u}^{2}+v^{2} r^{2} \dot{u}_{x}^{2}\right) d x=\int_{0}^{L} \frac{\rho}{2}\left(S \dot{u}^{2}+\nu^{2} I_{p}^{2} \dot{u}_{x}^{2}\right) d x \\
V & =\frac{1}{2} \int_{0}^{L}\left[\int_{S}\left(\sigma_{x x} \varepsilon_{x x}+\sigma_{r r} \varepsilon_{r r}+\sigma_{x r} \varepsilon_{x r}\right)\right] d x \\
& \cong \frac{1}{2} \int_{0}^{L} S E u_{x_{x}}^{2} d x .
\end{aligned}
$$

The contribution of the shear energy was neglected in the last expression, according to the Rayleigh-Love theory. The polar moment of inertia for a circular cross-section is $I_{p}=\pi R^{4} / 2$. The Lagrangian is $L=T-V=\int_{0}^{L} \Lambda d x$ in which the Lagrangian density $\Lambda$ is

$$
\Lambda=\frac{S}{2}\left(\rho \dot{u}^{2}+\rho v^{2} r_{g}^{2} \dot{u}_{x}^{2}-E u_{x}^{2}\right),
$$

with $r_{g}=R / \sqrt{2}$, which is the radius of gyration for a circular cross-section or radius $R$. The Lagrangian density must satisfy the Euler-Lagrange differential equation [14]:

$$
\frac{\partial}{\partial t}\left(\frac{\partial \Lambda}{\partial \dot{u}}\right)+\frac{\partial}{\partial x}\left(\frac{\partial \Lambda}{\partial u_{x}}\right)-\frac{\partial^{2}}{\partial x \partial t}\left(\frac{\partial \Lambda}{\partial \dot{u}_{x}}\right)=0,
$$

which leads to the differential equation of motion:

$$
\rho \ddot{u}-E u_{x x}-\rho \nu^{2} r_{g}^{2} \ddot{u}_{x x}=0 .
$$

This theory presented in [1] proved to be more accurate in predicting the variation of the longitudinal wave velocity with the frequency than the classical theory, especially for the high frequency range, in which we are interested. The classical formulation used in any textbook of longitudinal vibrations of cylindrical rods (e.g., $[15,16]$, etc.), can be recovered by neglecting the last term.

A harmonic plane wave propagating with velocity $c$, wavenumber $k=\omega / c$, and angular frequency $\omega=2 \pi f$, in which $f$ is the frequency, as

$$
u(x, t)=A \exp [i(k x-\omega t)]
$$

can be injected in (8) to get

$$
\left[-\rho \omega^{2}+k^{2} E-\rho \nu^{2} r_{g}^{2} \omega^{2} k^{2}\right] u(x, t)=0 .
$$

The dispersion equation for an elastic rod according to the Rayleigh-Love theory is then

$$
k^{2}\left(E-\rho v^{2} r_{g}^{2} \omega^{2}\right)=\rho \omega^{2} .
$$




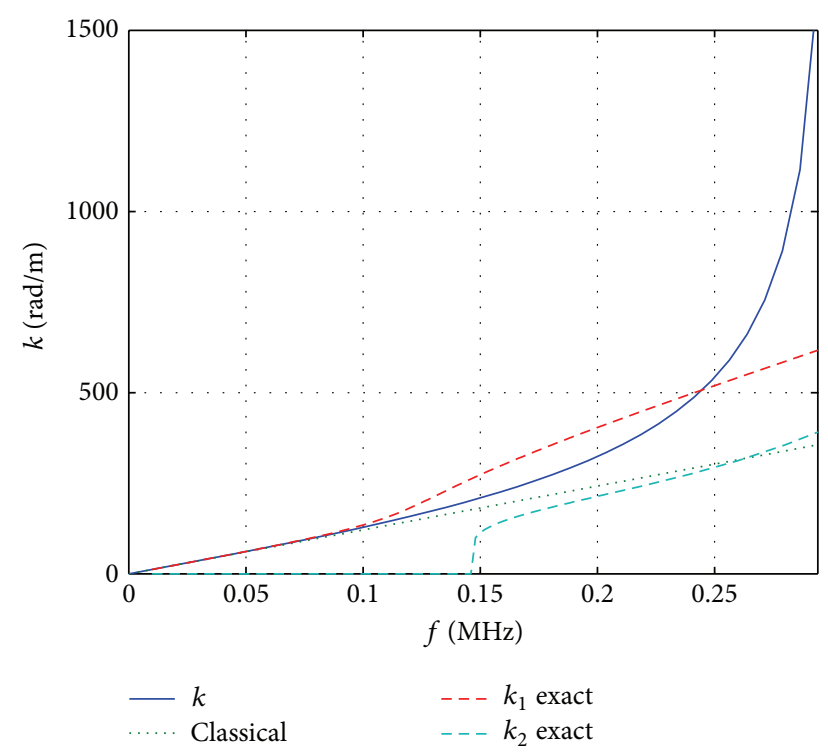

FIGURE 2: Dispersion curve for a rod using the Rayleigh-Love theory, compared to the classical and exact theories.

The dispersion curve can be plotted in several ways. Considering a rod of steel $(E=210 \mathrm{GPa}, \nu=0.31$, and $\rho=$ $7.8 \mathrm{~g} / \mathrm{cm}^{3}$ ) of radius $R=12.5 \mathrm{~mm}$, the dispersion curves in physical quantities are shown on Figure 2. For comparison, the classical model solution is also plotted, which is a straight line, as follows:

$$
k=\frac{\omega}{c_{0}} ; \quad c_{0}=\sqrt{\frac{E}{\rho}}
$$

and the first two exact solutions of the Pochhammer-Chree dispersion equation for an elastic cylinder [17]. Solving the exact solution is however not a very simple task, involving Bessel functions of complex arguments.

The Pochhammer-Chree dispersion equation is considerably more complicated than (8) of the Rayleigh-Love model, which is a reason for which simpler models to be pursued by many authors. We have developed a numerical root-finding algorithm for the Pochhammer-Chree dispersion equation, and the obtained wavenumbers of the first two modes are used for comparison against the approximate theories. These are called "exact," in the following, since they correspond to the "exact" elasticity theory but are obtained by numerical procedures with finite accuracy.

At relatively low frequencies (below $0.1 \mathrm{MHz}$ in this case) the Rayleigh-Love, classical, and first exact modes are coinciding. Between 0.1 and $0.25 \mathrm{MHz}$, the Rayleigh-Love solution is better than the classical solution, being closer to the exact longitudinal mode, called $L(0,1)$ of the exact solution. However, the Rayleigh-Love model has a major shortcoming for high frequencies: the wave number becomes purely imaginary, if

$$
f>\frac{1}{2 \pi} \sqrt{\frac{E}{\rho v^{2} r_{g}^{2}}}
$$

which means that $f<300 \mathrm{kHz}$ in this case. The RayleighLove model is thus inadequate for longitudinal vibrations in the $\mathrm{MHz}$ range.

2.2. The Rayleigh-Bishop Model. The Rayleigh-Bishop model is also a unimodal model, meaning that only one longitudinal wave mode is approximated. The kinetic energy $T$ and the strain potential energy $V$ are as follows:

$$
\begin{aligned}
T & =\int_{0}^{L} \frac{\rho}{2} \int_{S}\left(\dot{u}^{2}+v^{2} r^{2} \dot{u}_{x}^{2}\right) d x=\int_{0}^{L} \frac{\rho}{2}\left(S \dot{u}^{2}+v^{2} I_{p}^{2} \dot{u}_{x}^{2}\right) d x, \\
V & =\frac{1}{2} \int_{0}^{L}\left[\int_{S}\left(\sigma_{x x} \varepsilon_{x x}+\sigma_{r r} \varepsilon_{r r}+\sigma_{x r} \varepsilon_{x r}\right)\right] d x \\
& \cong \frac{1}{2} \int_{0}^{L}\left[S E u_{x}^{2}+v^{2} \mu I_{p} u_{x x}^{2}\right] d x .
\end{aligned}
$$

The Lagrangian density $\Lambda$ is

$$
\Lambda=\frac{S}{2}\left(\rho \dot{u}^{2}+\rho v^{2} r_{g}^{2} \dot{u}_{x}^{2}-E u_{x}^{2}-v^{2} \mu r_{g}^{2} u_{x x}^{2}\right),
$$

in which $r_{g}=R / \sqrt{2}$ is the radius of gyration for a circular cross section or radius $R$.

The Lagrangian density must satisfy [14] the EulerLagrange differential equation:

$$
\begin{gathered}
\frac{\partial}{\partial t}\left(\frac{\partial \Lambda}{\partial \dot{u}}\right)+\frac{\partial}{\partial x}\left(\frac{\partial \Lambda}{\partial u_{x}}\right)-\frac{\partial^{2}}{\partial x \partial t}\left(\frac{\partial \Lambda}{\partial \dot{u}_{x}}\right) \\
-\frac{\partial^{2}}{\partial x^{2}}\left(\frac{\partial \Lambda}{\partial u_{, x x}}\right)=0,
\end{gathered}
$$

which leads to the differential equation of motion:

$$
\rho \ddot{u}-E u_{x x}-\rho v^{2} r_{g}^{2} \ddot{u}_{x x}+v^{2} \mu r_{g}^{2} u_{x x x x}=0 .
$$

A harmonic plane wave solution can be also used in this case:

$$
u(x, t)=A \exp [i(k x-\omega t)] .
$$

Equation (17) becomes

$$
\left[-\rho \omega^{2}+k^{2} E-\rho \nu^{2} r_{g}^{2} \omega^{2} k^{2}+\nu^{2} \mu r_{g}^{2} k^{4}\right] u(x, t)=0 .
$$

The dispersion equation for an elastic rod according to the Rayleigh-Bishop theory is then

$$
k^{2}\left(E-\rho v^{2} r_{g}^{2} \omega^{2}+\nu^{2} \mu r_{g}^{2} k^{2}\right)=\rho \omega^{2} .
$$

The same rod is used in the numerical simulation and the results from our numerical analysis are shown on Figure 3. The model proves to be considerably more accurate than the classical model (dotted line). The dispersion curve of the Rayleigh-Bishop model tends towards the first exact mode of the Pochhammer-Chree theory in case of low frequencies and towards the second mode as the frequencies increase. There is no critical frequency, beyond which there are no solutions, as in the Rayleigh-Love model, so that a wavenumber $k$ can be obtained for any frequency. This model is better adapted to model high frequency longitudinal vibrations, as required for ultrasonic transducers. 


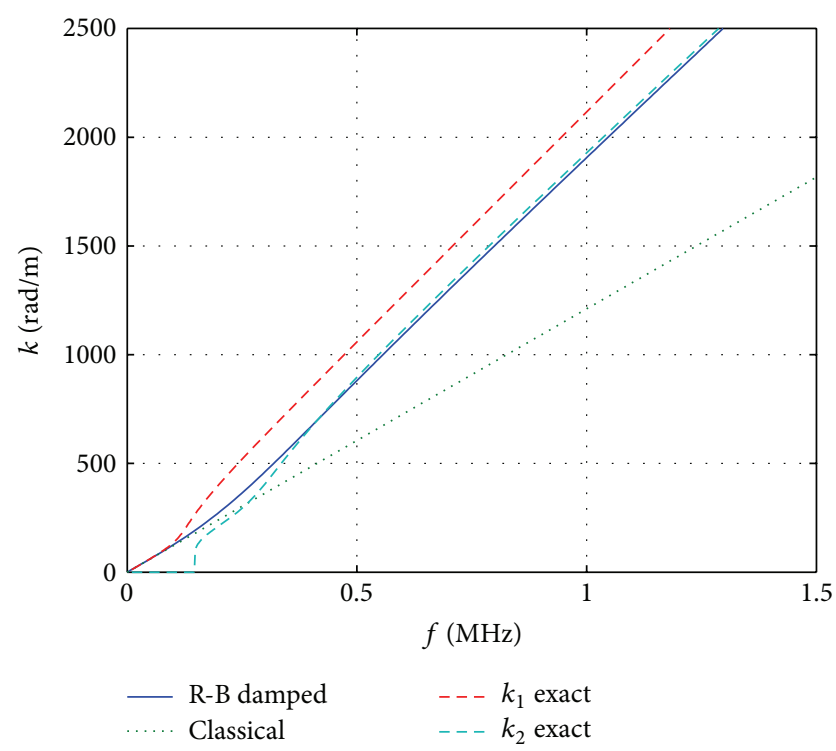

FIGURE 3: Dispersion curve for a rod using the Rayleigh-Bishop theory, compared to the classical and exact theories.

\section{Damping of Longitudinal Vibrations}

As shown above, the Rayleigh-Bishop unimodal model is the best in approximating the dispersion curves. The vibrations are always subjected to damping. In this paper, we are including damping, which is assumed to be proportional to the strain velocity variation of the selected model. Under this assumption, using a proportionality damping constant $\beta$, the differential equation of motion (17) becomes

$$
\rho \ddot{u}-E u_{x x}-\rho v^{2} r_{g}^{2} \ddot{u}_{x x}+v^{2} \mu r_{g}^{2} u,_{x x x x}-\beta E \dot{u}_{x x}=0 .
$$

Looking for damped vibrations of rods, one can select the displacement function as follows:

$$
u(x, t)=A \exp (i k x) \exp (-i \omega t),
$$

in which the angular frequency is expected to be complex valued. The dispersion equation becomes

$$
k^{2}\left(E-\rho \nu^{2} r_{g}^{2} \omega^{2}+\nu^{2} \mu r_{g}^{2} k^{2}-i \beta \omega\right)=\rho \omega^{2} .
$$

For a given real wavenumber $k$, the complex angular frequency can be obtained as $\omega=\omega_{r}+i \omega_{i}$, by solving a simple polynomial equation of third degree, for which numerical and even closed form solutions exist.

The real and imaginary parts of the angular frequency as functions of the real wavenumber, for the same cylindrical rod are shown on Figures 4 and 5, respectively. The influence on the real part of the angular frequency is negligible for the selected frequency range, as can be seen in Figure 4. For values of $\beta$ up to $10^{4} \mathrm{~kg} \cdot \mathrm{s}^{-1} \cdot \mathrm{m}^{-1}$, the influence on damping, represented by the imaginary part, is less than $3 \%$ of the real part, but, for $\beta=10^{5} \mathrm{~kg} \cdot \mathrm{s}^{-1} \cdot \mathrm{m}^{-1}$, the imaginary part is increasing up to between 15 and $30 \%$ of the real part. Damping is increasing in absolute value with frequency, but, for increasing frequency, the imaginary part tends to a eliminated value depending on $\beta$.

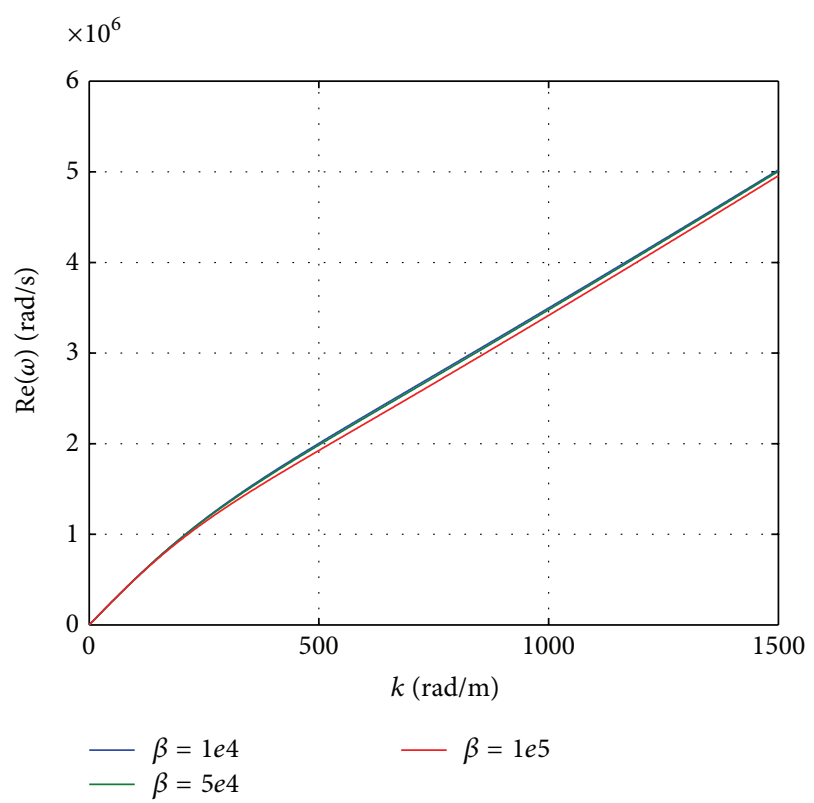

FIGURE 4: Angular frequency (real part) versus frequency for three damping coefficients $\beta$.

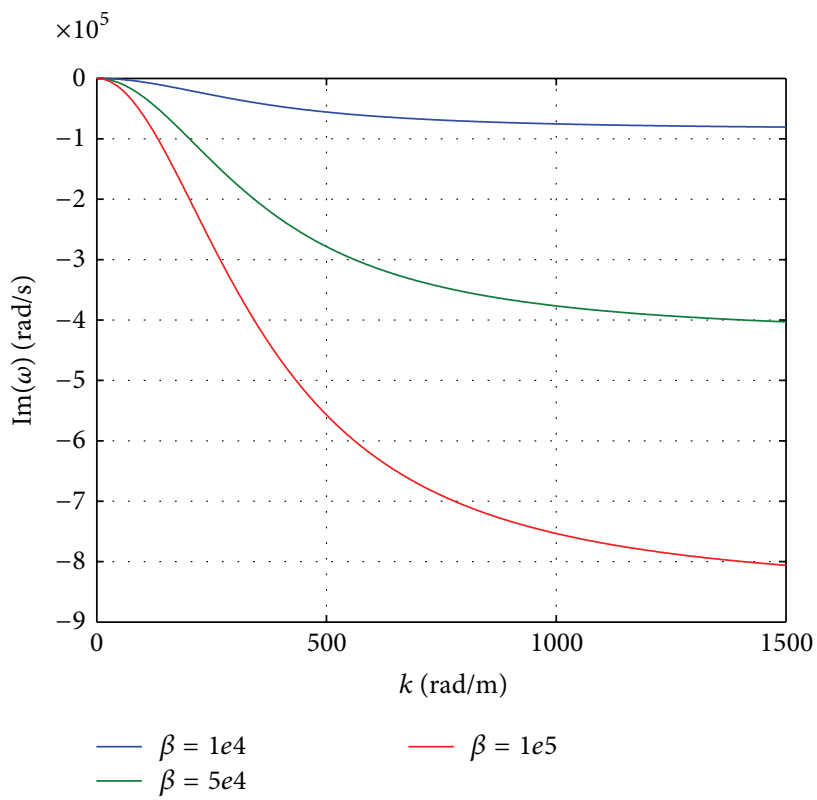

FIGURE 5: Angular frequency (imaginary part) versus frequency for three damping coefficients $\beta$.

\section{Longitudinal Damped Vibrations of a Short Rod}

The foreseen application of the longitudinal vibrations is in ultrasonic transducers. An analytic model based on the Rayleigh-Bishop model without damping was presented in [18]. The damped vibrations mechanical model of such a transducer, based on the formulation of the previous section, 


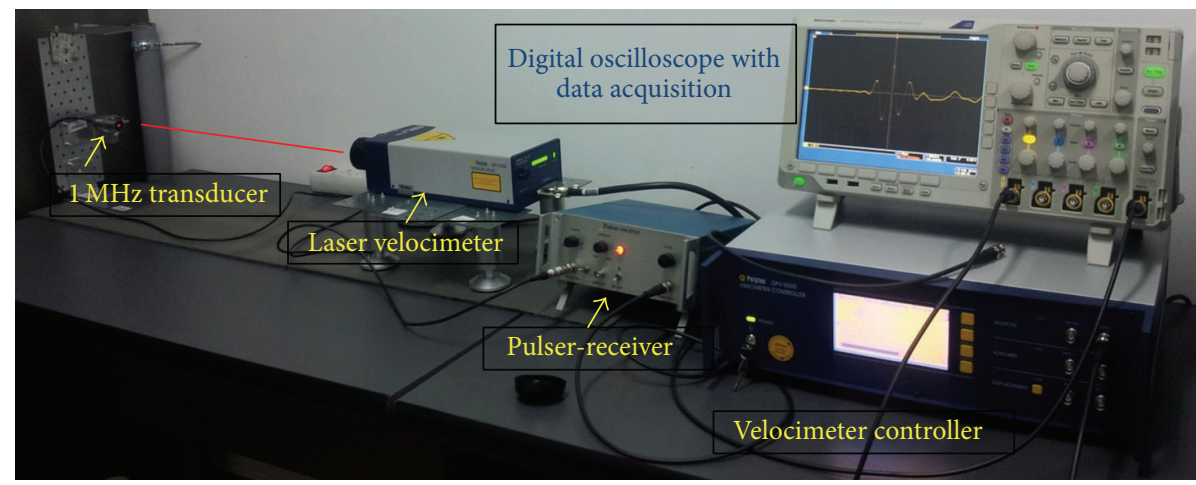

FIGURE 6: Experimental setup used to measure free response of the ultrasonic transducer.

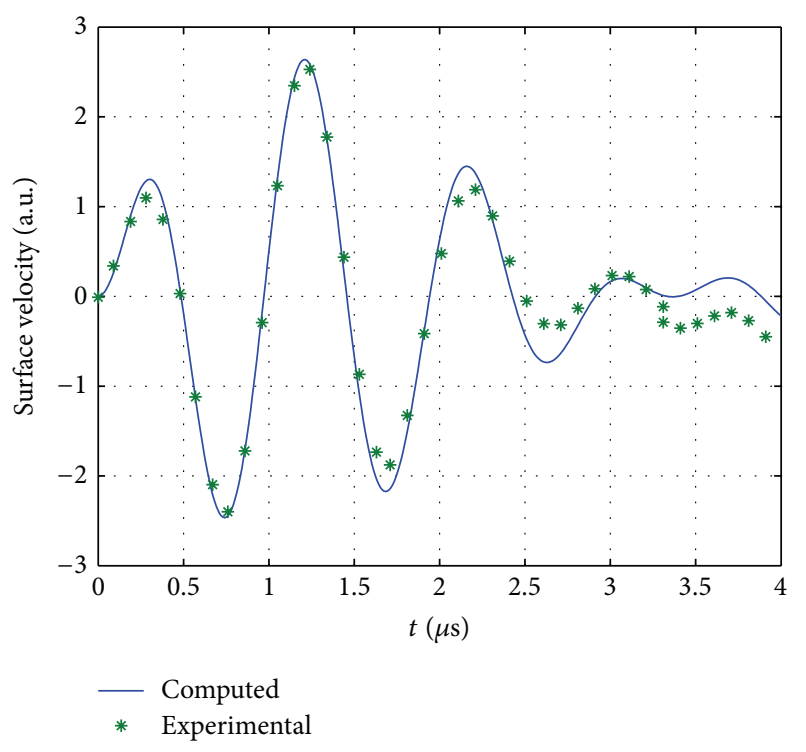

FIGURE 7: Computed data superposed on the measured normal velocity on transducer surface.

can be represented as two coaxial rods of identical diameter connected by the piezoelectric crystal, as in [15].

We consider now two connected rods, made of different materials $E_{1}, E_{2}$, cross sections $S_{1}$ and $S_{2}$, and lengths $L_{1}$ and $L_{2}$, in order to follow the derivation of equivalent equations in Section 2.3 of [15]. A practical particular case will be deduced for $E_{1}=E_{2}$ and $S_{1}=S_{2}$, joined at $x=0$ (Figure 1(b)).

The damped harmonic motion in the two rods can be expressed as

$$
\begin{array}{r}
u_{m}(x, t)=\left[A_{m} \cos \left(k_{m} x\right)+B_{m} \sin \left(k_{m} x\right)\right] \exp \left(-i \omega_{m} t\right) \\
m=1,2,
\end{array}
$$

in which the complex angular frequencies $\omega_{m}$ are solutions of (23) for given wavenumbers $k_{m}$. The boundary conditions for the first rod are represented by the longitudinal displacement and stress continuity at $x=0$ and longitudinal stress cancelling at $x=L_{1}$ :

$$
\begin{gathered}
u_{1}(0, t)=u_{2}(0, t) ; \quad E_{1} S_{1} u_{1, x}(0, t)=E_{2} S_{2} u_{2, x}(0, t) ; \\
u_{1, x}\left(L_{1}, t\right)=0 .
\end{gathered}
$$

For the second rod, the longitudinal stress cancels at the free end

$$
u_{2, x}\left(-L_{2}, t\right)=0 \text {. }
$$

The exact boundary conditions for the free end in the Rayleigh-Bishop case can be found in [1]. Here, the shear stress is not taken into account. These four equations can be written as

$$
\begin{gathered}
A_{1} \exp \left(-i \omega_{1} t\right)=A_{2} \exp \left(-i \omega_{2} t\right), \\
E_{1} S_{1} k_{1} B_{1} \exp \left(-i \omega_{1} t\right)=E_{2} S_{2} k_{2} B_{2} \exp \left(-i \omega_{2} t\right), \\
k_{1}\left[-A_{1} \sin \left(k_{1} L_{1}\right)+B_{1} \cos \left(k_{1} L_{1}\right)\right]=0, \\
k_{2}\left[A_{2} \sin \left(k_{1} L_{1}\right)+B_{2} \cos \left(-k_{2} L_{2}\right)\right]=0 .
\end{gathered}
$$

It follows that

$$
\begin{gathered}
\frac{A_{1}}{A_{2}}=\frac{B_{2}}{B_{1}} \frac{k_{2} E_{2} S_{2}}{k_{1} E_{1} S_{1}} ; \quad \frac{B_{1}}{A_{1}}=\tan \left(k_{1} L_{1}\right) ; \\
\frac{B_{2}}{A_{2}}=-\tan \left(k_{2} L_{2}\right)
\end{gathered}
$$

or

$$
\frac{k_{2} E_{2} S_{2}}{k_{1} E_{1} S_{1}} \sin \left(k_{2} L_{2}\right) \cos \left(k_{1} L_{1}\right)+\sin \left(k_{1} L_{1}\right) \cos \left(k_{2} L_{2}\right)=0 \text {. }
$$

This equation resembles (2.3.16) from [15], except for the ratio of wavenumbers, which is missing by mistake in the cited reference. In the particular case of identical materials and cross sections, a relation between the two wavenumbers can be thus obtained. In practical cases of ultrasonic transducers, the first antisymmetric mode of vibration, with respect to 
$x=0$, is used. This means that the condition $\cos \left(k_{1} L_{1}\right)=$ 0 will provide the wavenumber for the first rod and, from (29), it follows that $\cos \left(k_{2} L_{2}\right)=0$ provides the smallest wavenumber for the second rod. Consequently,

$$
k_{1}=\frac{\pi}{2 L_{1}} ; \quad k_{2}=\frac{\pi}{2 L_{2}}
$$

If the two rods have different lengths, according to (23), the assembly will have two resonance frequencies and two damping ratios. For $\mathrm{MHz}$ range frequencies, the cylinders lengths are less than $1 \mathrm{~mm}$ for the considered steel.

\section{Experimental Validation}

A $1 \mathrm{MHz}$ central frequency longitudinal wave ultrasonic transducer has received a short pulse of $200 \mathrm{~V}$ from the pulser-receiver. The free response of the transducer was measured by a laser velocimeter and recorded on a digital oscilloscope (Figure 6). The simplest model of the transducer can be imagined as two connected short steel rods, according to the previous section. The material choice is made in order to minimize acoustic impedance mismatch in case of nondestructive testing of steel materials.

By iterative testing of rod lengths and attenuations, the following parameters have been obtained for a best fit of the recorded normal velocity of the transducer: $L_{1}=0.84 \mathrm{~mm}$ and $L_{2}=0.64 \mathrm{~mm}$ and attenuation: $\operatorname{Imag}(\omega)=-6 e 5 \mathrm{rad} / \mathrm{s}$.

The agreement (Figure 7) is satisfactory for most applications, proving the adequacy of the developed simple and efficient mechanical model.

\section{Conclusions}

Longitudinal vibrations of rods at high frequencies, in the $\mathrm{MHz}$ range, have been investigated using the classical and the Rayleigh-Love models, which both proved inadequate since the dispersion curves are too far from the exact values or simply constants in this frequency range. The Rayleigh-Bishop model was adopted and we have introduced a damping term in this model. As a consequence of this improved model, wavenumbers are almost unaffected by damping. On the other hand, the wave damping is increasing with frequency in an asymptotic manner, which can be accepted in a simple model.

A free vibrating ultrasonic transducer was modeled as two unequal rods acted by negligible thin piezoelectric element between them. The purpose is to mimic the behavior of the real transducer by the simplest mechanical model. By an iterative procedure, the optimal choice of parameters has led to an accurate representation of measured normal velocity of a $1 \mathrm{MHz}$ longitudinal ultrasonic transducer.

\section{Conflict of Interests}

The authors declare that there is no conflict of interests regarding the publication of this paper.

\section{Acknowledgment}

The present work was supported by the Romanian UEFISCDI Exploratory Research Project PN-II-ID-PCE-2011-3-0512.

\section{References}

[1] M. Shatalov, J. Marais, I. Fedotov, M. D. Tenkam, and M. Schmidt, "Longitudinal vibration of isotropic solid rods: from classical to modern theories," in Advances in Computer Science and Engineering, M. Schmidt, Ed., pp. 187-214, InTech, 2011.

[2] I. Fedotov, J. Marais, M. Shatalov, and H. M. Tenkam, "Hyperbolic models arising in the theory of longitudinal vibration of elastic bars," The Australian Journal of Mathematical Analysis and Applications, vol. 7, no. 2, pp. 1-18, 2011.

[3] J. E. M. Medina, F. Buiochi, and J. Adamowski, "Numerical modeling of a circular piezoelectric ultrasonic transducer radiating in water," in Proceedings of the ABCM Symposium Series in Mechatronics, vol. 2, pp. 458-464, 2006.

[4] N. N. Abboud, G. L. Wojcik, D. K. Vaughan, J. Mould, D. J. Powell, and L. Nikodym, "Finite element modeling for ultrasonic transducers," in International Symposium on Medical Imaging, Ultrasonic Transducer Engineering Conference, vol. 3341 of Proceedings of SPIE, pp. 19-42, San Diego, Calif, USA, February 1998.

[5] M. Ali, "Analysis of broadband piezoelectric transducers by discrete time model," Egyptian Journal of Solids, vol. 23, no. 2, pp. 287-295, 2000.

[6] R. Guelaz, D. Kourtiche, M. Nadi, and Y. Herve, "Ultrasonic piezoceramic transducer modeling with VHDL-AMS IEEE 1076.1," in Proceedings of the 3rd IEEE Conference on Sensors, pp. 87-90, Vienna, Austria, October 2004.

[7] M. Dragan, R. Milan, P. Zoran, and S. Goran, "Influence of ultrasonic transducer acoustic impedances and dimensions on its input electrical impedance," Working and Living Environmental Protection, vol. 5, no. 1, pp. 59-72, 2008.

[8] M. W. Nygren, Finite Element Modeling of Piezoelectric Ultrasonic Transducers, Norwegian University of Science and Technology, Trondheim, Norway, 2011.

[9] J. Kocbach, Finite Element Modeling of Ultrasonic Piezoelectric Transducers, Department of Physics, University of Bergen, Bergen, Norway, 2000.

[10] D. Porto, Design Methodology and Numerical Optimization of Ultrasonic Transducers for Spinal Surgery, École Polytechnique Fédérale de Lausanne, Lausanne, Switzerland, 2009.

[11] F. Schubert and B. Lamek, "3-D ultrasonic transducer modeling using the elastodynamic finite integration technique in combination with point-source-synthesis," in Proceedings of 4th International Workshop NDT in Progress, Prague, Czech Republic, November 2007.

[12] R. Pintelon, P. Guillaume, S. Vanlanduit, K. de Belder, and Y. Rolain, "Identification of Young's modulus from broadband modal analysis experiments," Mechanical Systems and Signal Processing, vol. 18, no. 4, pp. 699-726, 2004.

[13] M. Castaings, C. Bacon, B. Hosten, and M. V. Predoi, "Finite element predictions for the dynamic response of thermoviscoelasicity material structures," Journal of the Acoustical Society of America, vol. 115, no. 3, pp. 1125-1133, 2004.

[14] R. Courant and D. Hilbert, Methods of Mathematical Physics, John Wiley \& Sons, New York, NY, USA, 1953.

[15] K. F. Graff, Wave Motion in Elastic Solids, Dover, New York, NY, USA, 1975. 
[16] C. W. de Silva, Vibration. Fundamentals and Practice, CRC Press LLC, Boca Raton, Fla, USA, 2000.

[17] J. D. Achenbach, Wave Propagation in Elastic Solids, North Holland, Amsterdam, The Netherlands, 1973.

[18] H. Tenkam, "A theory of piezoelectric thickness vibrator transducer based on the Rayleigh and Bishop model," in Proceedings of the World Congress on Engineering (WCE '12), vol. 1, p. 48, London, UK, July 2012. 

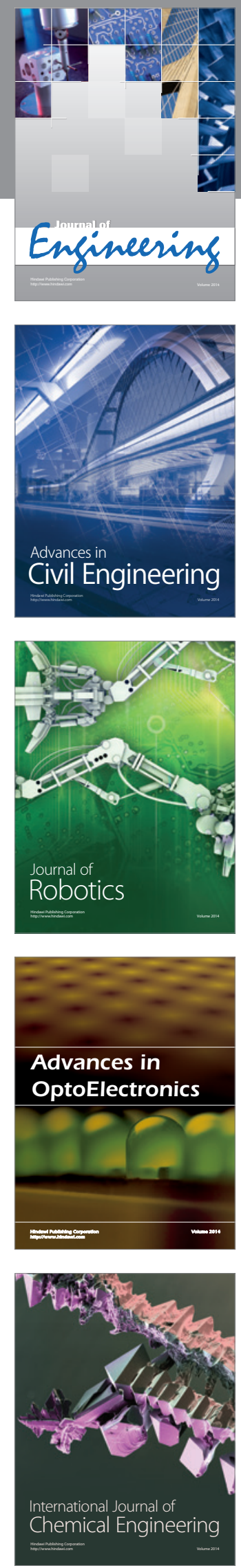

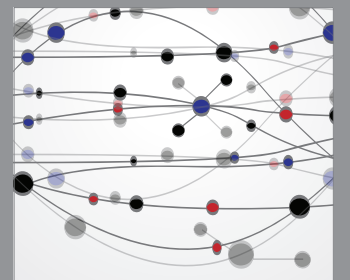

The Scientific World Journal
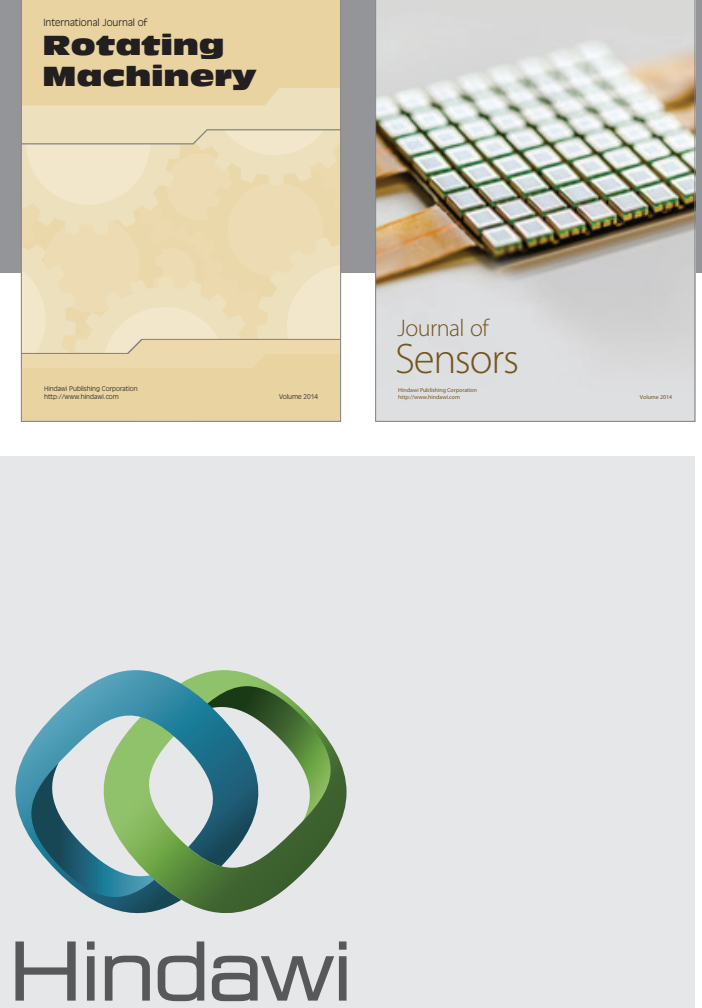

Submit your manuscripts at http://www.hindawi.com
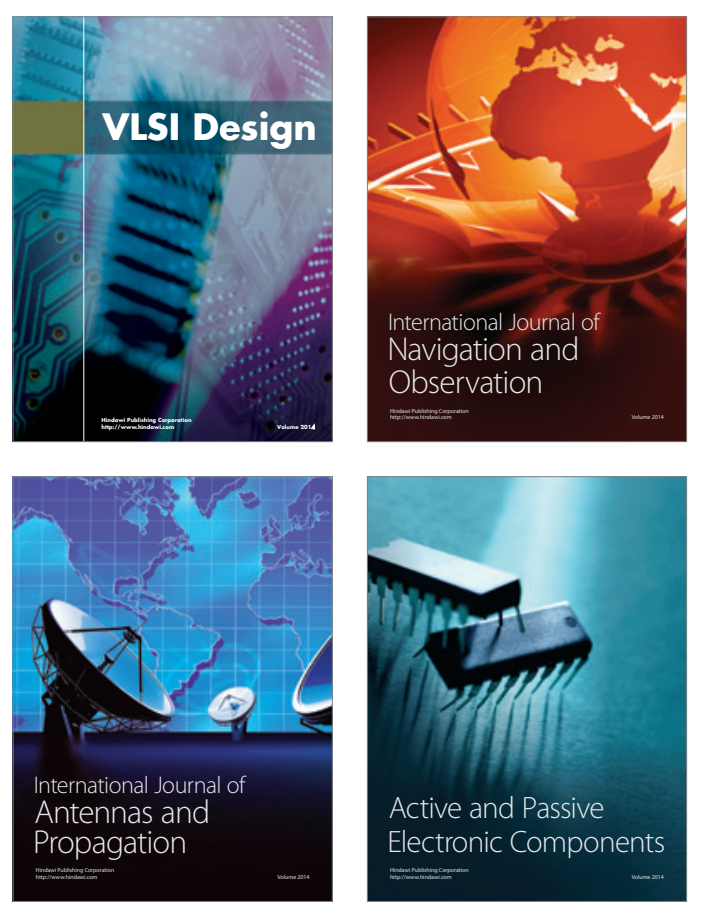
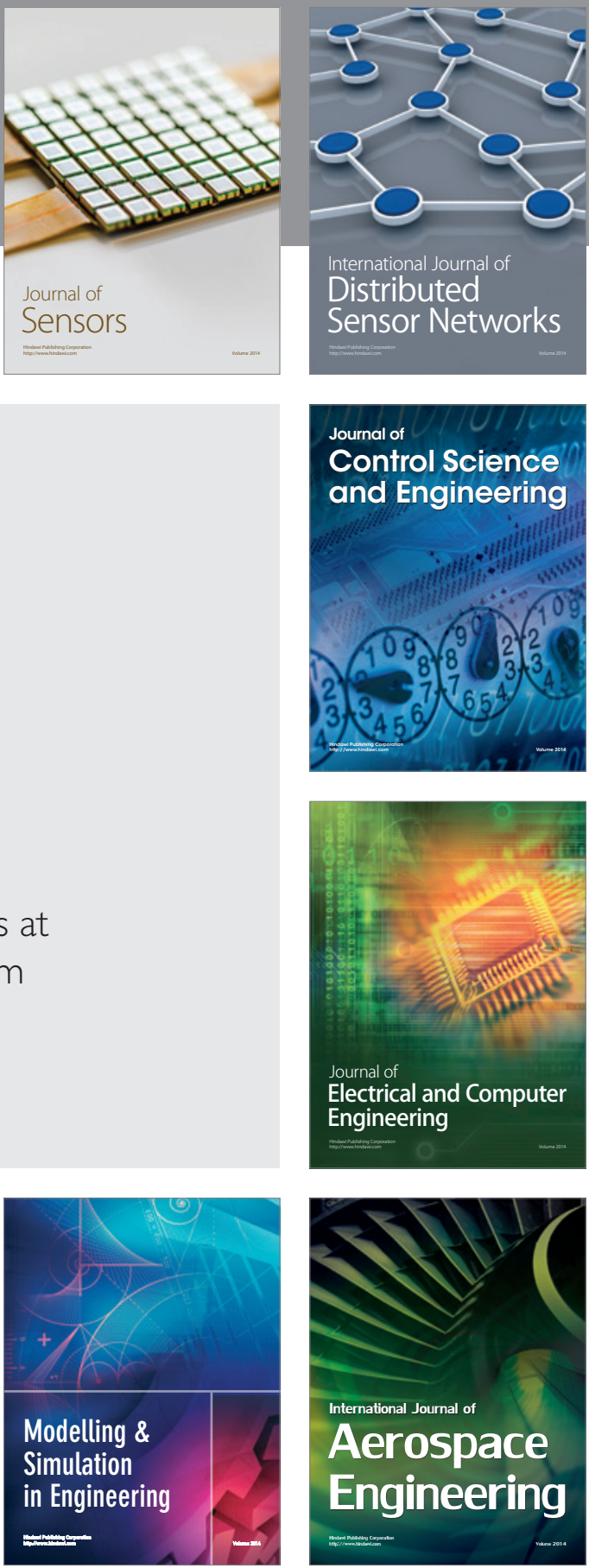

Journal of

Control Science

and Engineering
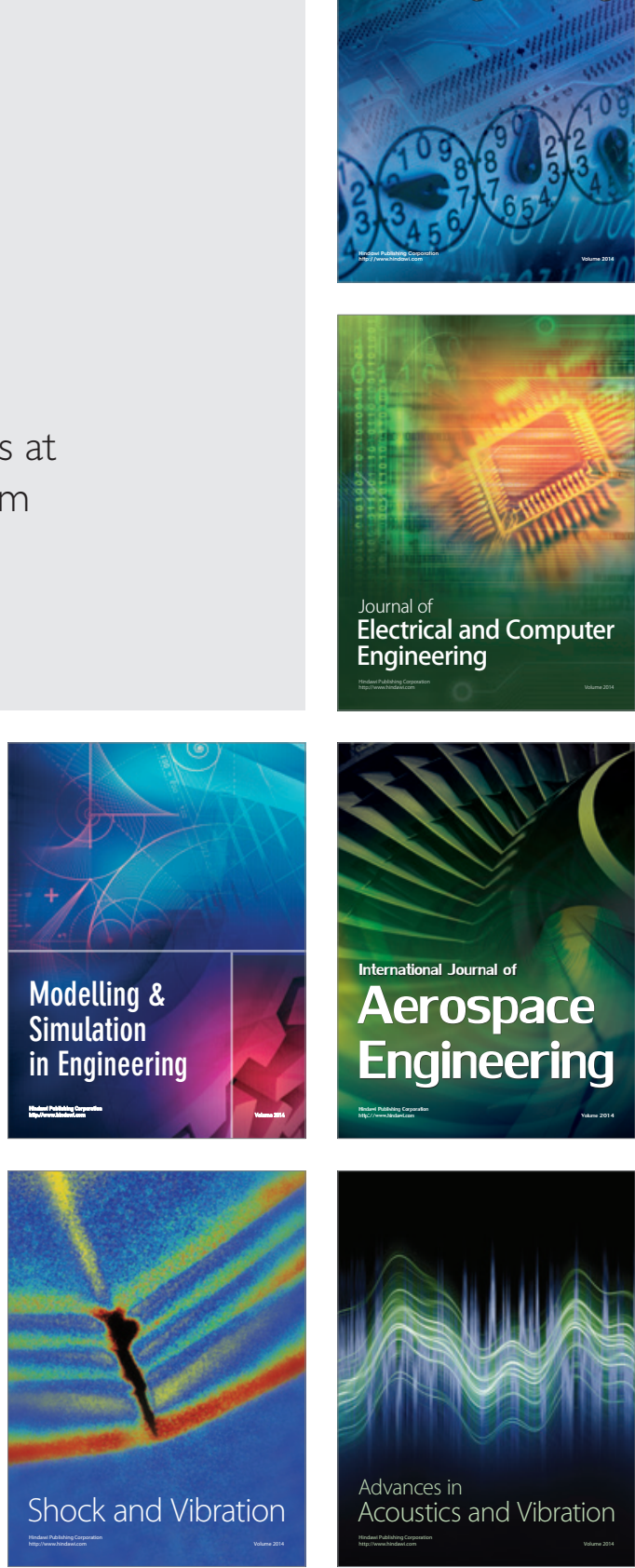